\title{
Taking Your iPad 2 to the Max
}

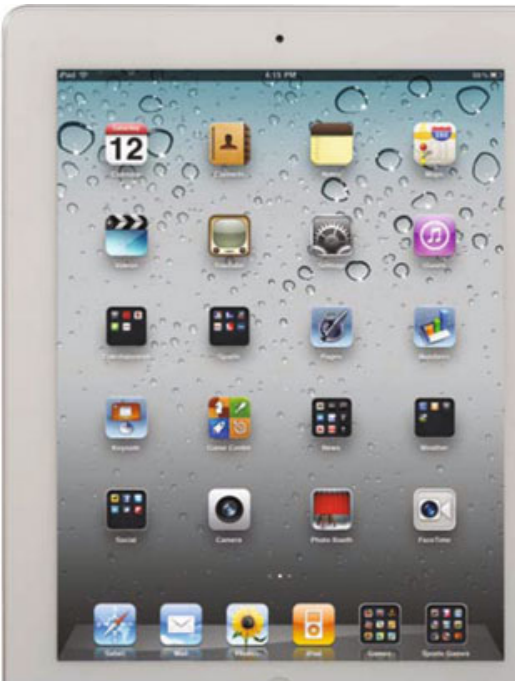

Erica Sadun

Michael Grothaus

Steve Sande 


\section{Taking Your iPad 2 to the Max}

Copyright ( 2011 by Erica Sadun, Michael Grothaus, and Steve Sande

All rights reserved. No part of this work may be reproduced or transmitted in any form or by any means, electronic or mechanical, including photocopying, recording, or by any information storage or retrieval system, without the prior written permission of the copyright owner and the publisher.

ISBN 978-1-4302-3539-2

ISBN 978-1-4302-3540-8 (eBook)

Trademarked names, logos, and images may appear in this book. Rather than use a trademark symbol with every occurrence of a trademarked name, logo, or image we use the names, logos, and images only in an editorial fashion and to the benefit of the trademark owner, with no intention of infringement of the trademark.

The use in this publication of trade names, trademarks, service marks, and similar terms, even if they are not identified as such, is not to be taken as an expression of opinion as to whether or not they are subject to proprietary rights.

President and Publisher: Paul Manning

Lead Editor: Michelle Lowman

Developmental Editor: Douglas Pundick

Technical Reviewer: Dave Caolo

Editorial Board: Steve Anglin, Mark Beckner, Ewan Buckingham, Gary Cornell, Jonathan Gennick, Jonathan Hassell, Michelle Lowman, Matthew Moodie, Jeff Olson, Jeffrey Pepper, Frank Pohlmann, Douglas Pundick, Ben Renow-Clarke, Dominic Shakeshaft, Matt Wade, Tom Welsh

Coordinating Editor: Kelly Moritz

Copy Editors: Tracy Brown, William McManus, and Patrick Meador

Compositor: MacPS, LLC

Indexer: Toma Mulligan

Artist: April Milne

Cover Designer: Anna Ishchenko

Distributed to the book trade worldwide by Springer Science+Business Media, LLC., 233 Spring Street, 6th Floor, New York, NY 10013. Phone 1-800-SPRINGER, fax (201) 348-4505, e-mail orders-ny@springer-sbm.com, or visitwww.springeronline.com.

For information on translations, please e-mail rights@apress.com, or visit www.apress.com.

Apress and friends of ED books may be purchased in bulk for academic, corporate, or promotional use. eBook versions and licenses are also available for most titles. For more information, reference our Special Bulk Sales-eBook Licensing web page at WwW.apress.com/bulk-sales.

The information in this book is distributed on an "as is" basis, without warranty. Although every precaution has been taken in the preparation of this work, neither the author(s) nor Apress shall have any liability to any person or entity with respect to any loss or damage caused or alleged to be caused directly or indirectly by the information contained in this work. 
To my sister, Crissy Grothaus, who I hope will buy an iPad before her daughter does.

$$
\text { - Michael }
$$

To Hayden Starr, for your friendship, for your unceasing hard work, and for being such an excellent (and patient!) teacher of history and geography to my kids.

$$
\text { -Erica }
$$

To my sweet old cat Ruby, who has spent many hours giving me inspiration by laying her head on the side of my iPad 2 and purring.

$$
\text { -Steve }
$$




\section{Contents at a Glance}

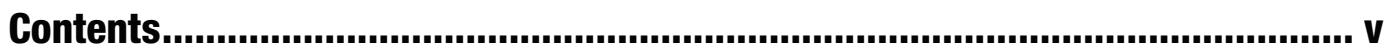

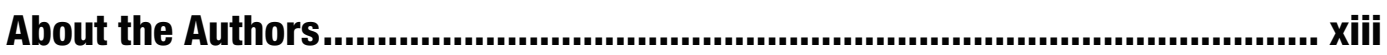

About the Technical Reviewer ...................................................................xiv

Acknowledgments ..........................................................................................XV

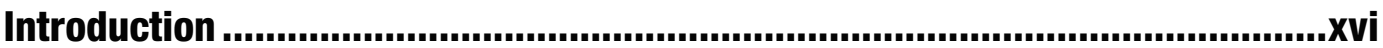

Chapter 1: Bringing Your iPad Home........................................................... 1

Chapter 2: Putting Your Data and Media on Your iPad............................... 19

Chapter 3: Exploring the iPad Hardware .................................................. 51

Chapter 4: Interacting with Your iPad.................................................... 71

Chapter 5: Connecting to the Internet ................................................... 107

Chapter 6: Browsing the Internet with Safari......................................... 123

Chapter 7: Touching Your Music and Video.............................................. 147

Chapter 8: Shopping for Apps, Books, Music, and More........................... 189

Chapter 9: Using iBooks ....................................................................... 213

Chapter 10: Leveraging Your Desk Set.................................................. 245

Chapter 11: Setting Up and Using Mail.................................................. 267

Chapter 12: Working with Maps ............................................................... 297

Chapter 13: Touching Your Digital Photos................................................ 327

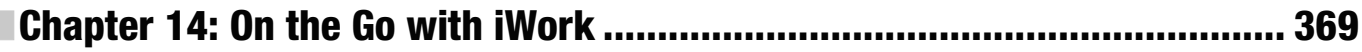

Chapter 15: Using the iPad Cameras with Camera,

Photobooth, and FaceTime .................................................... 401

Chapter 16: Other Great Ways to Use Your iPad...................................... 431

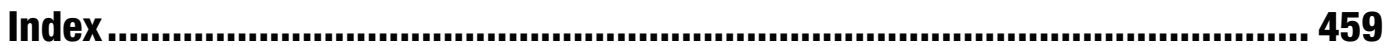




\section{Contents}

Contents at a Glance....................................................................................... iv

About the Authors................................................................................. xiii

About the Technical Reviewer ............................................................xiv

Acknowledgments ............................................................................xv

Introduction .....................................................................................................xvi

Chapter 1: Bringing Your iPad Home....................................................... 1

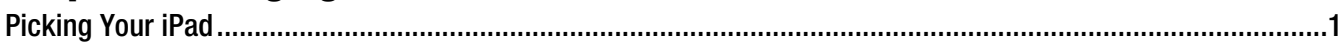

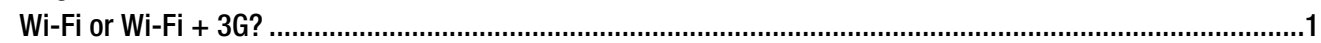

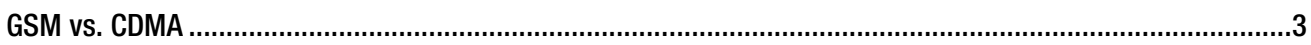

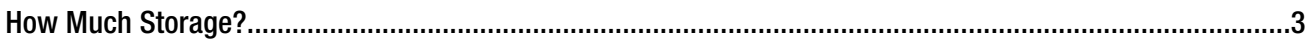

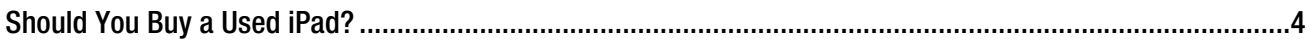

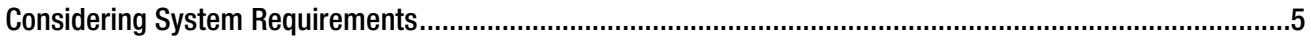

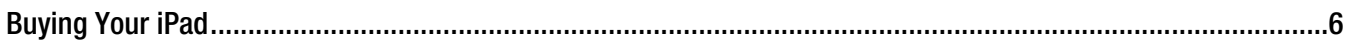

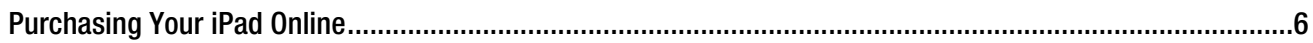

Repairs, Returns, Warranties, AppleCare, and Insurance ..................................................................

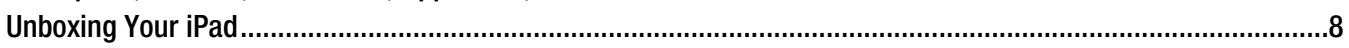

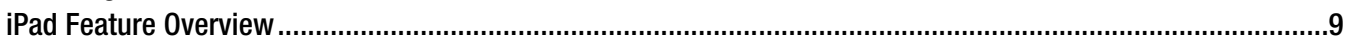

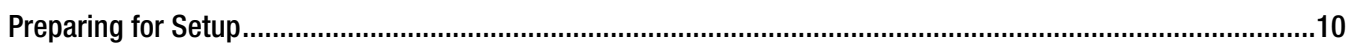

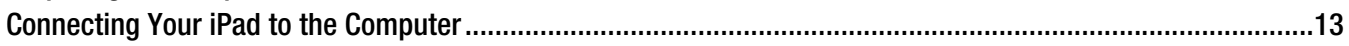

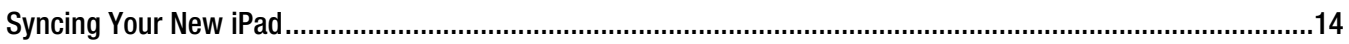

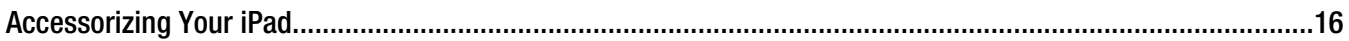

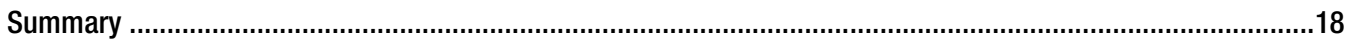

Chapter 2: Putting Your Data and Media on Your iPad................................ 19

Working with iTunes ...................................................................................................................19

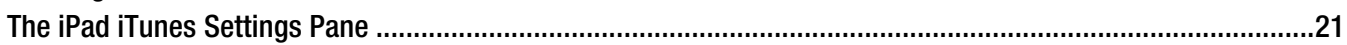

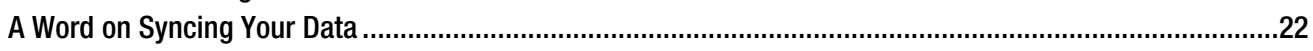

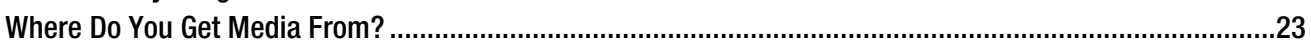

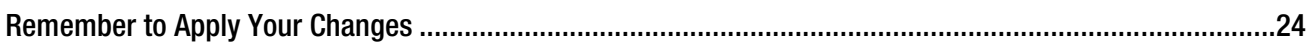

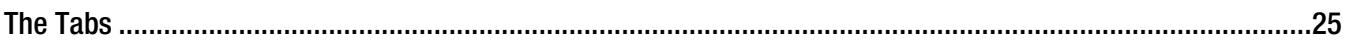

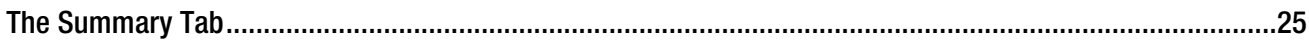

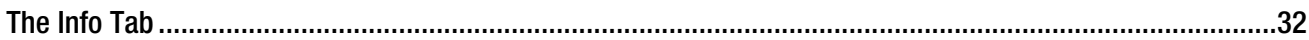




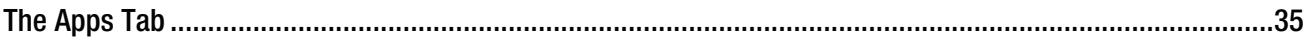

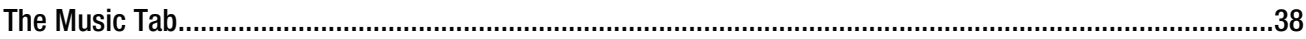

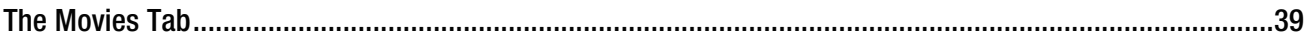

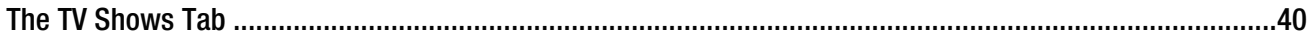

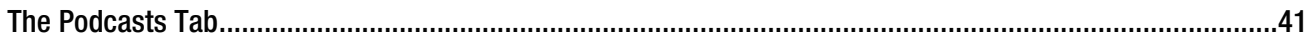

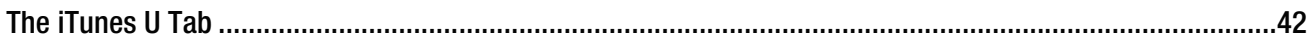

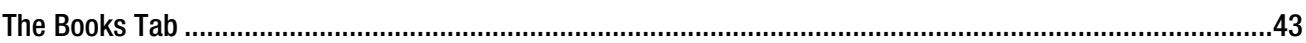

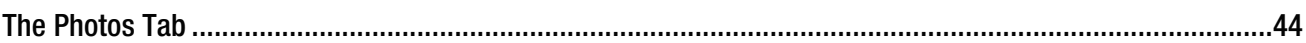

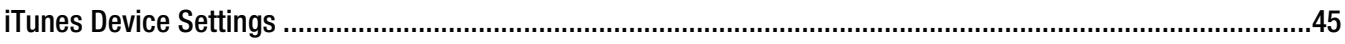

Restoring

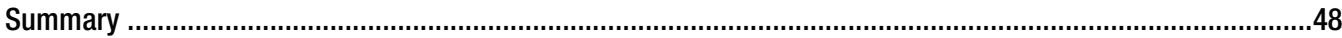

Chapter 3: Exploring the iPad Hardware ..................................................... 51

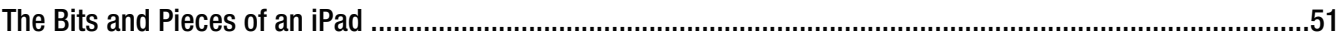

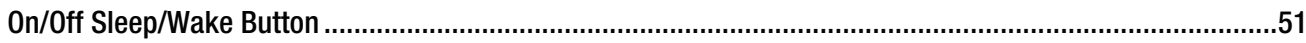

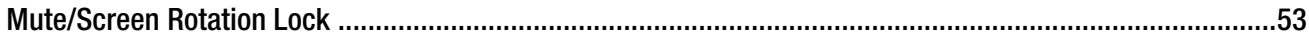

Volume Toggle

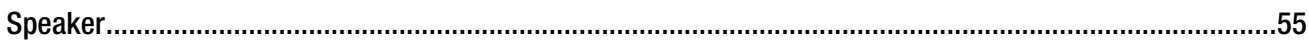

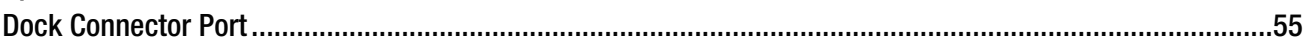

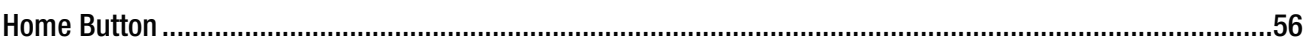

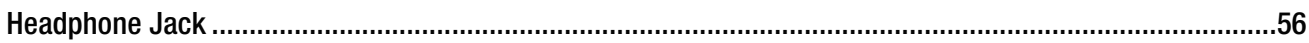

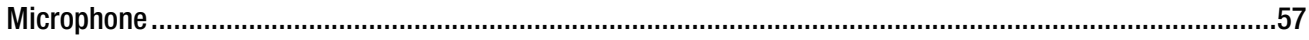

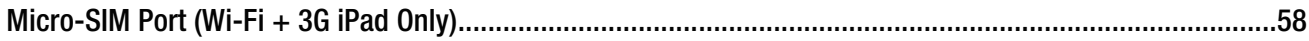

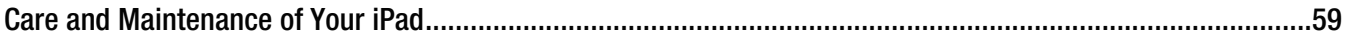

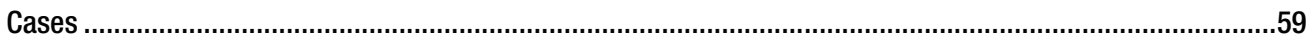

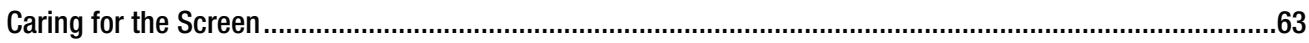

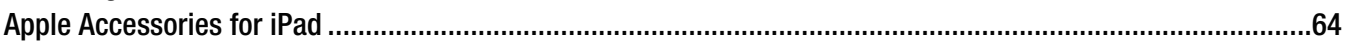

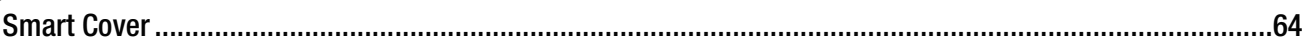

iPad 2 Dock

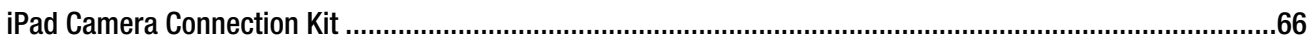

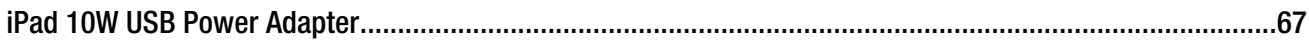

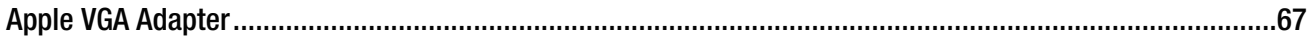

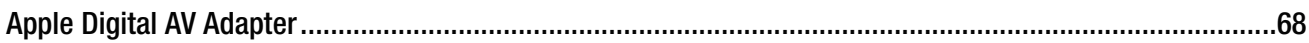

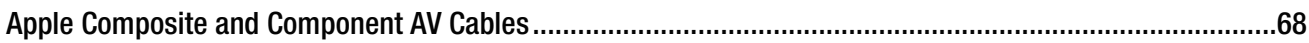

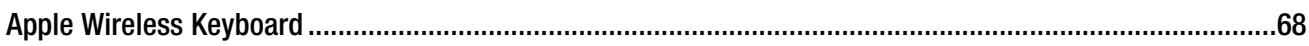

Apple Earphones with Remote and Mic ....................................................................................69

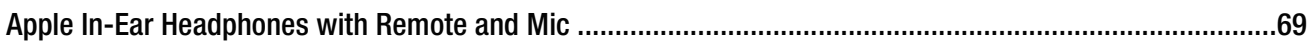

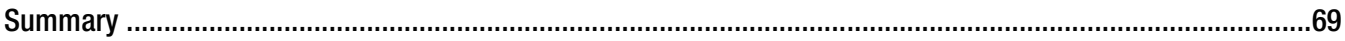

Chapter 4: Interacting with Your iPad .......................................................... 71

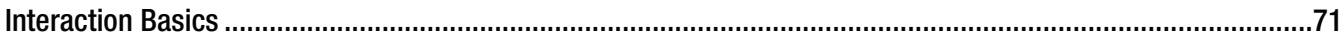

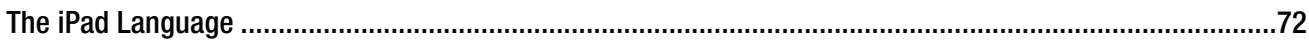

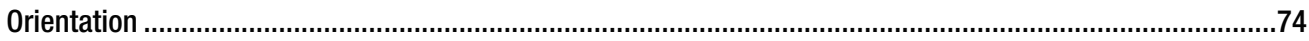

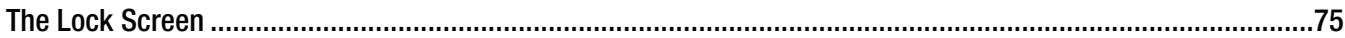

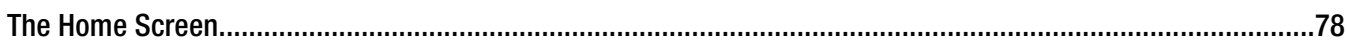

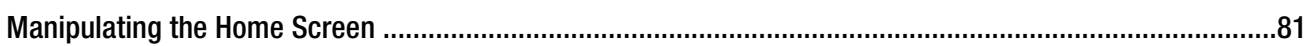

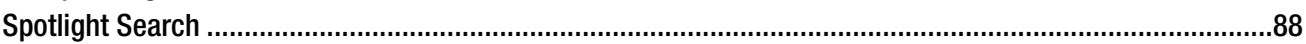

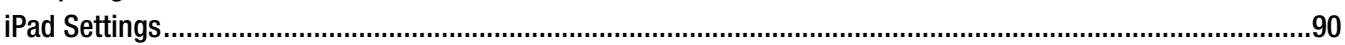

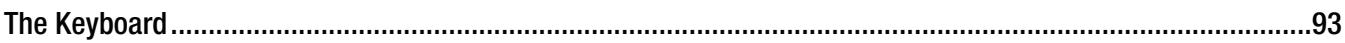




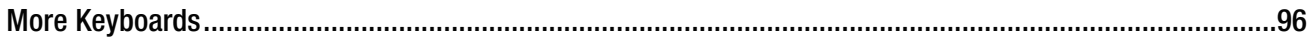

Getting Started

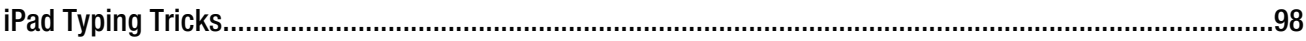

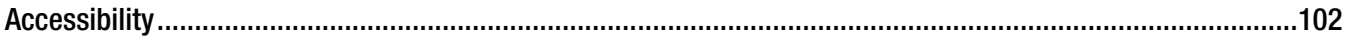

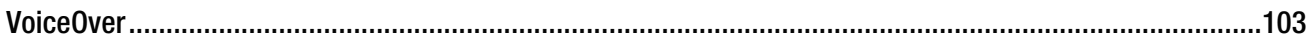

Zoom

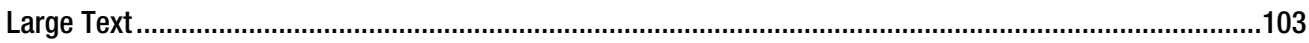

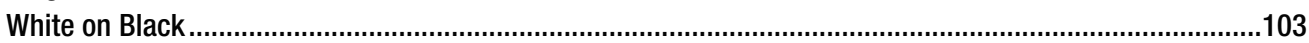

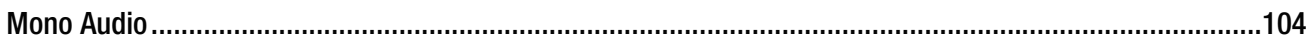

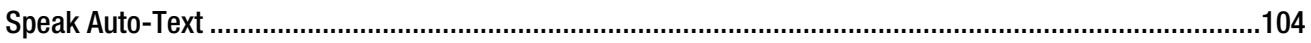

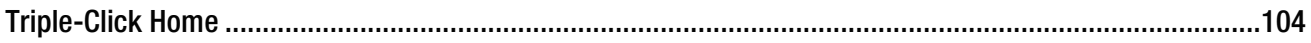

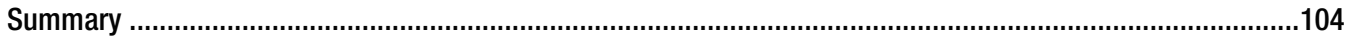

Chapter 5: Connecting to the Internet ................................................... 107

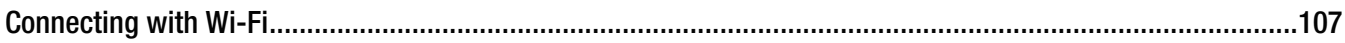

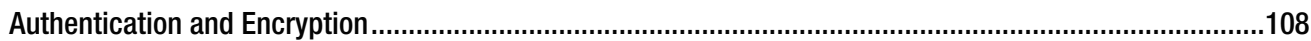

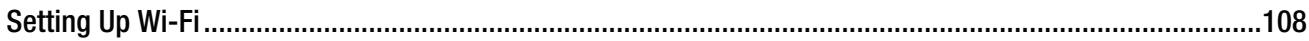

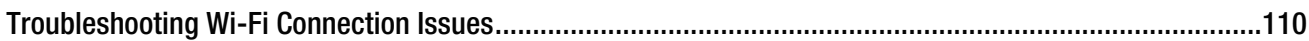

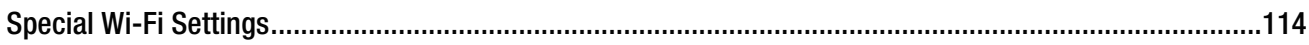

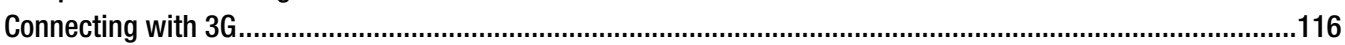

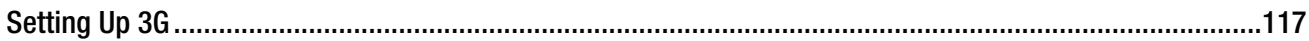

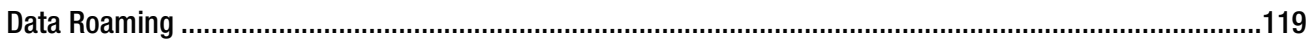

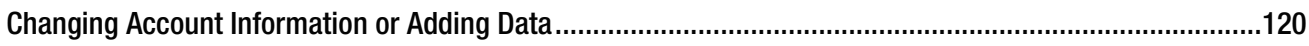

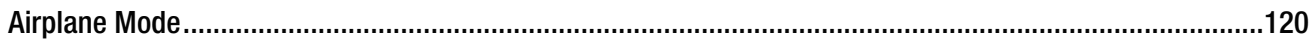

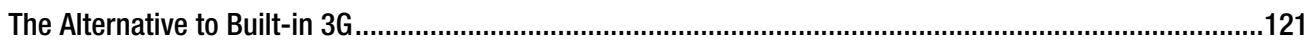

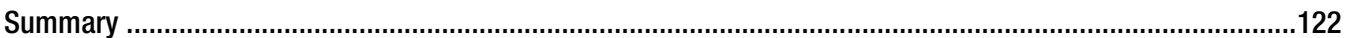

Chapter 6: Browsing the Internet with Safari.......................................... 123

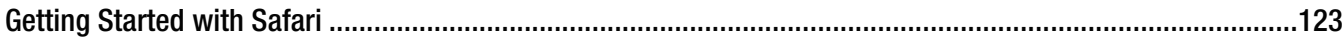

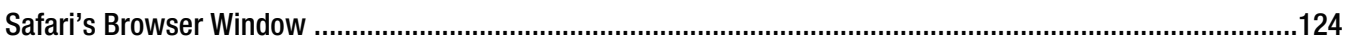

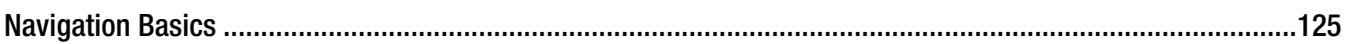

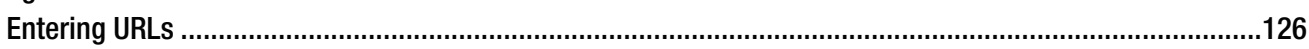

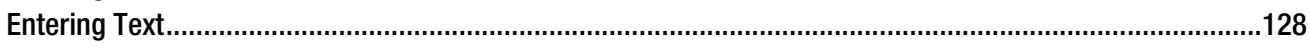

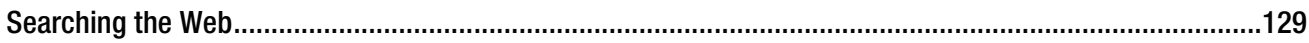

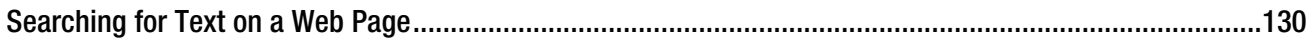

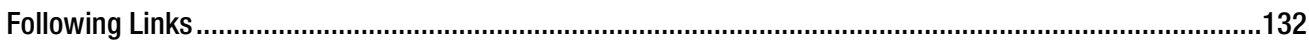

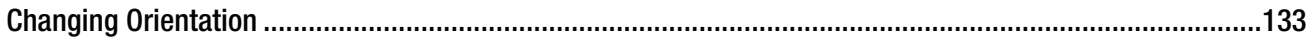

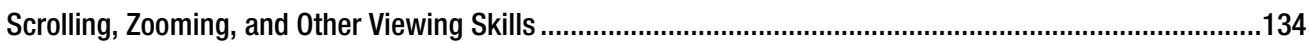

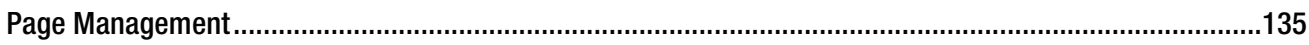

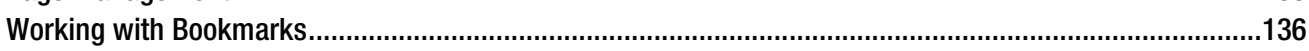

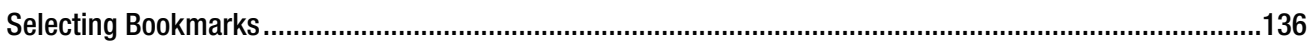

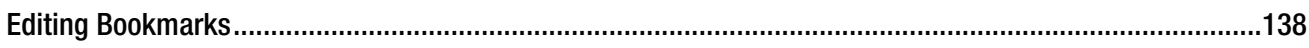

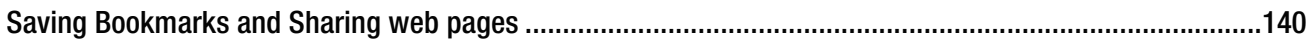

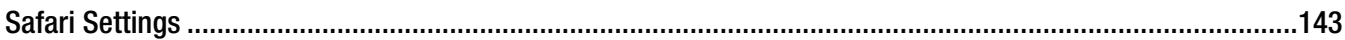

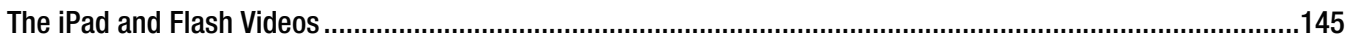

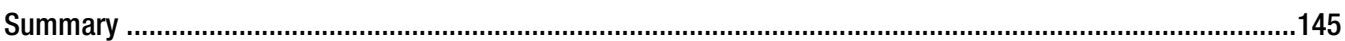

Chapter 7: Touching Your Music and Video............................................. 147

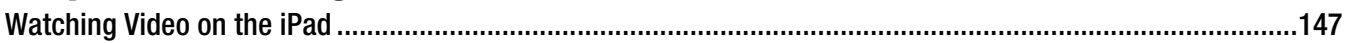

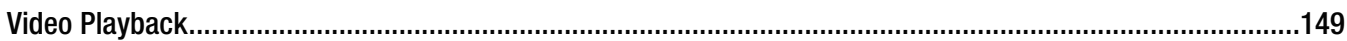


Videos App

Playing a Video

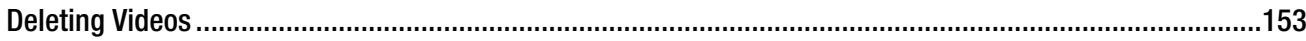

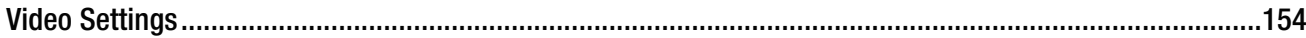

YouTube

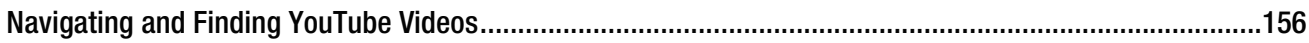

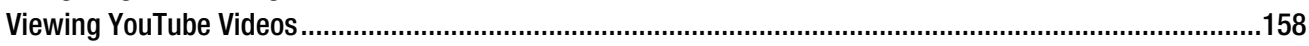

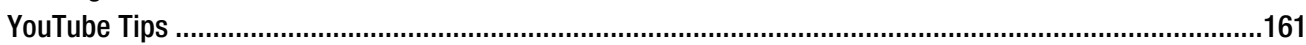

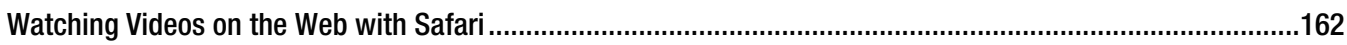

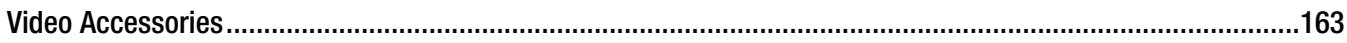

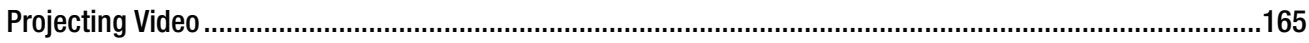

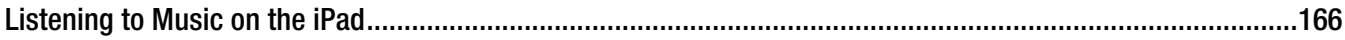

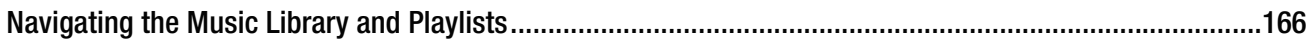

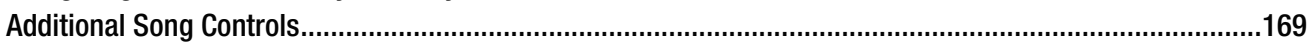

Playing Audio from the Music Library and Playlists......................................................................170

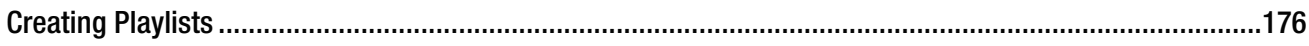

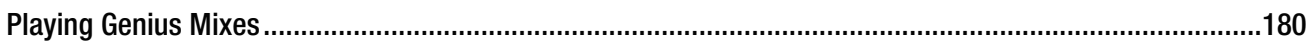

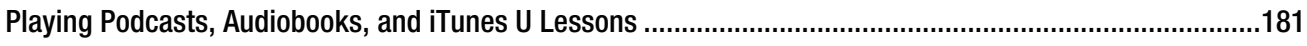

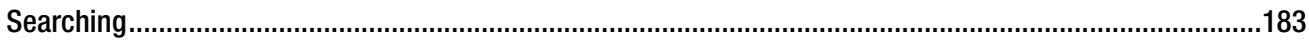

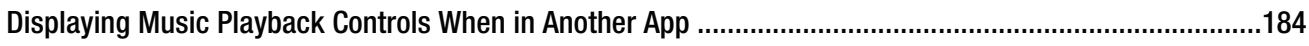

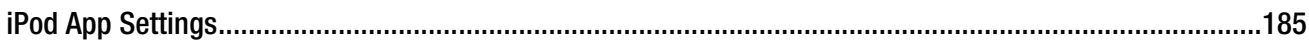

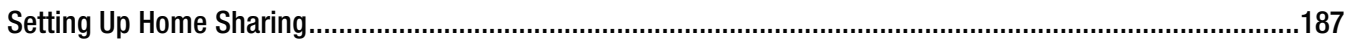

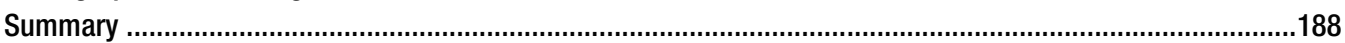

Chapter 8: Shopping for Apps, Books, Music, and More ............................. 189

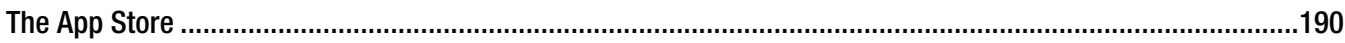

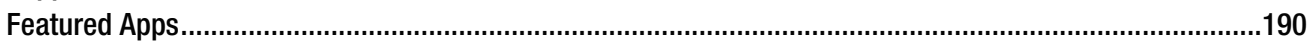

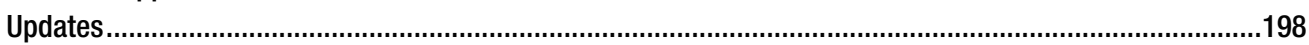

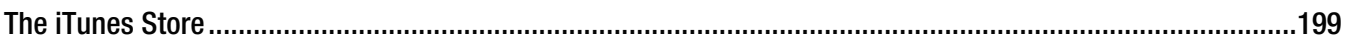

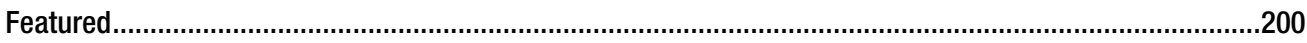

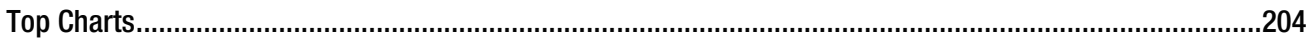

Genius

The iBookstore

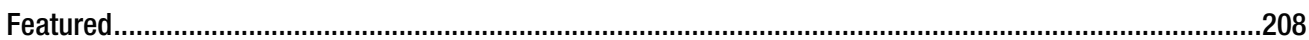

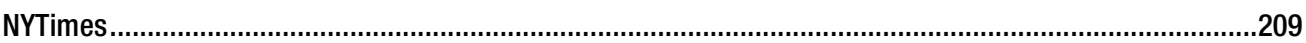

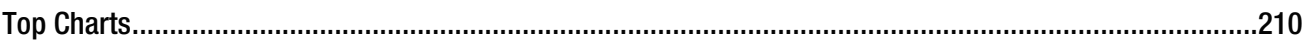

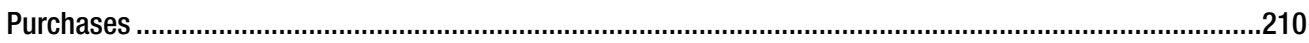

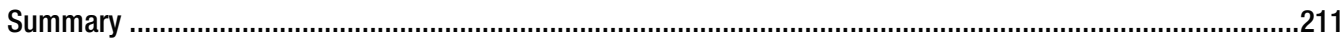

Chapter 9: Using iBooks .................................................................. 213

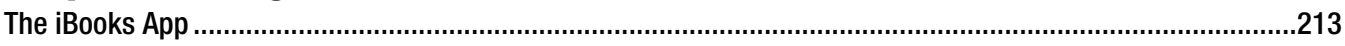

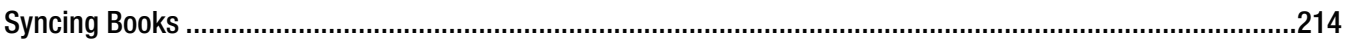

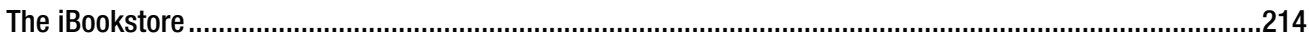

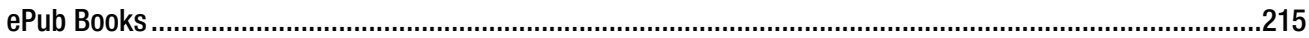

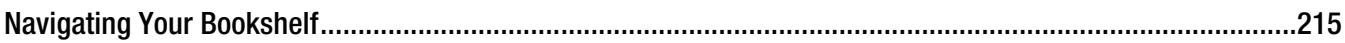

Organizing Your Books into Collections ............................................................................................218

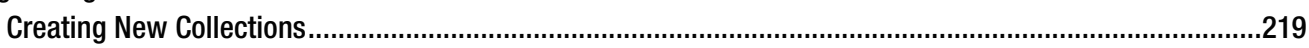

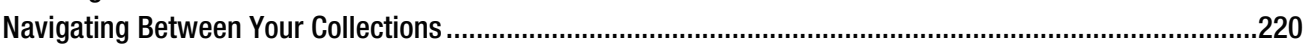

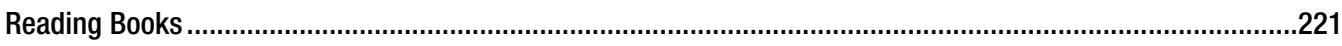




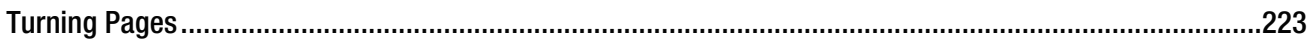

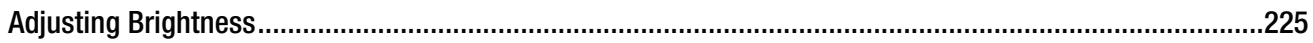

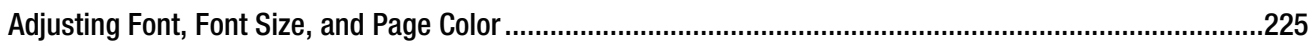

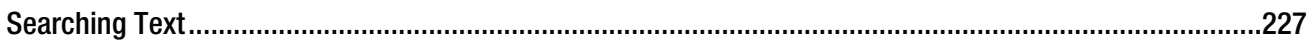

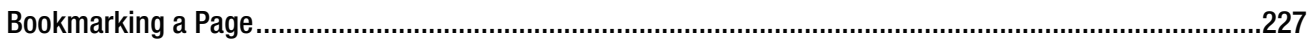

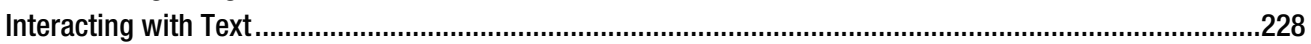

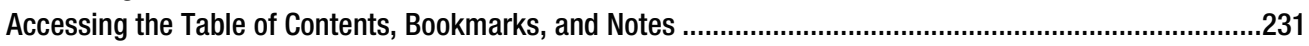

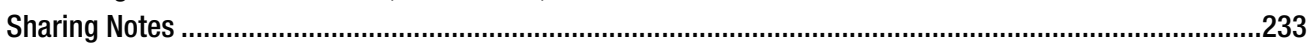

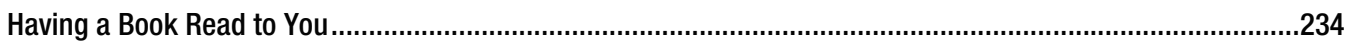

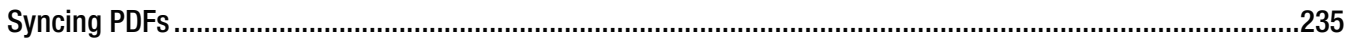

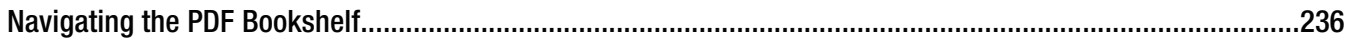

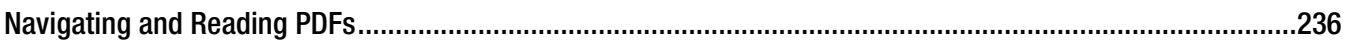

Using the Contact Sheet

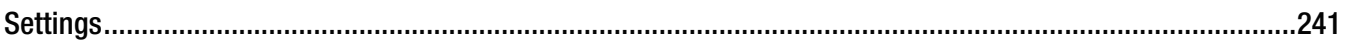

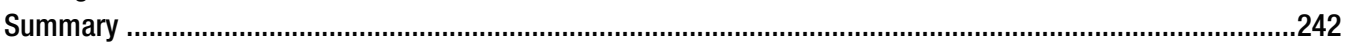

Chapter 10: Leveraging Your Desk Set..................................................... 245

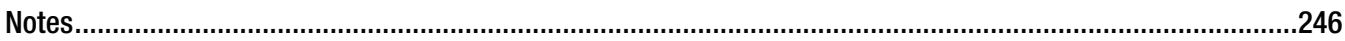

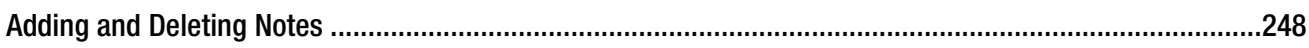

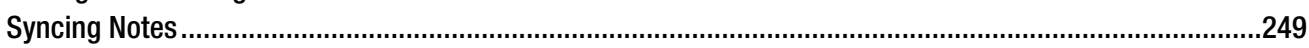

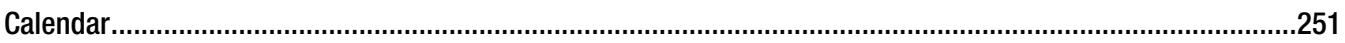

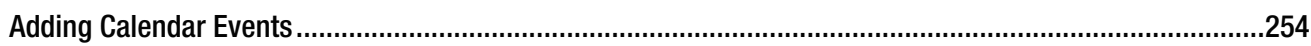

Syncing Calendar

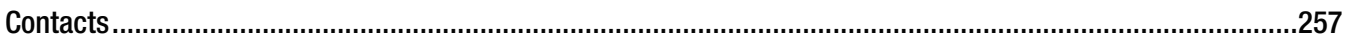

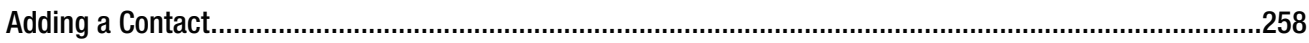

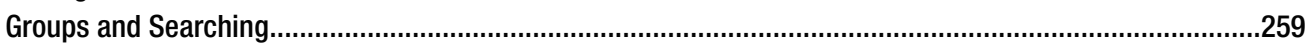

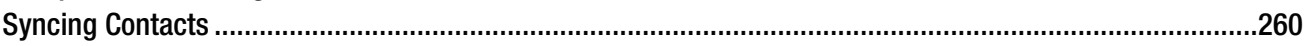

MobileMe Synchronization.................................................................................................................262

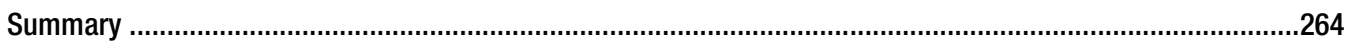

Chapter 11: Setting Up and Using Mail........................................................ 267

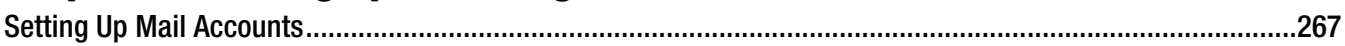

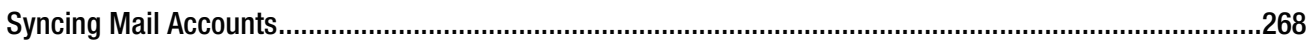

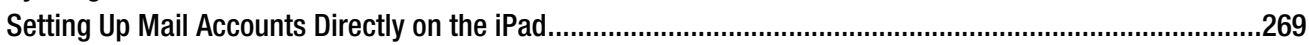

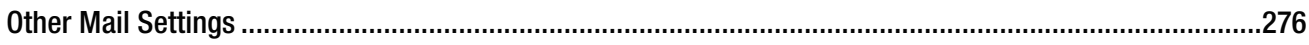

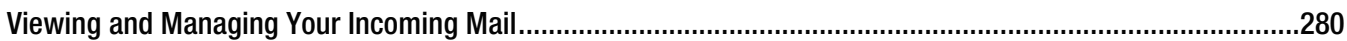

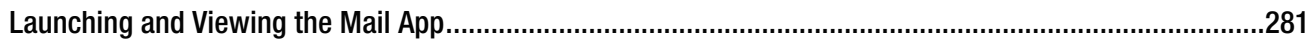

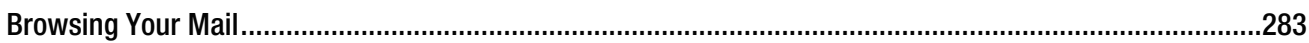

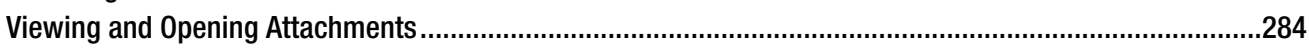

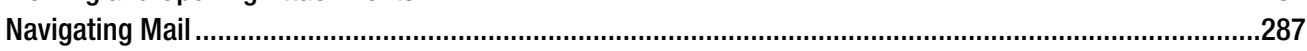

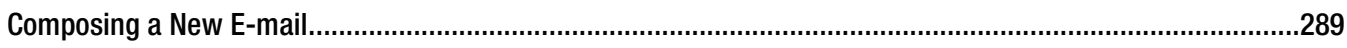

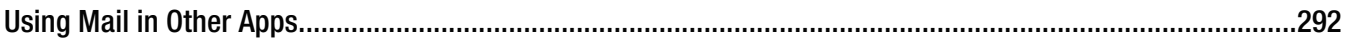

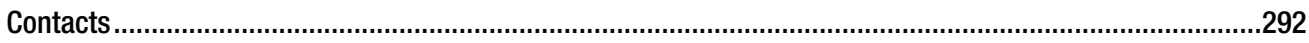

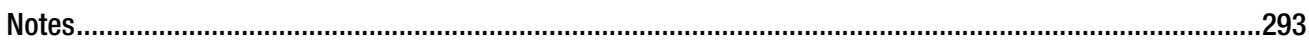

YouTube

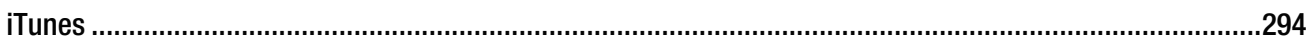

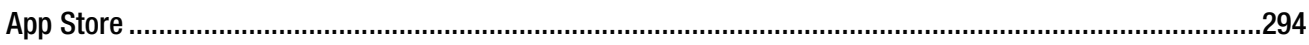

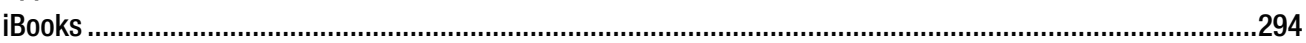

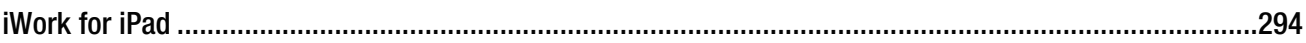


Photos

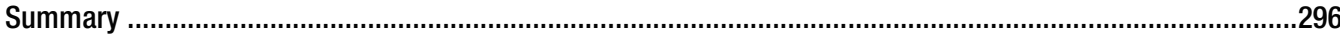

Chapter 12: Working with Maps ..................................................... 297

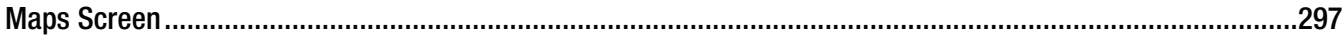

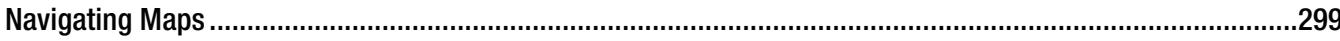

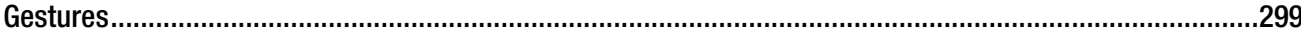

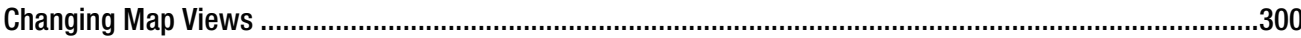

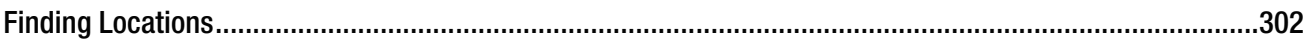

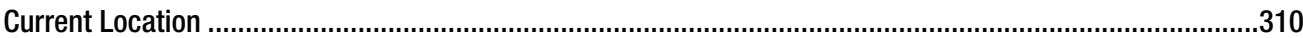

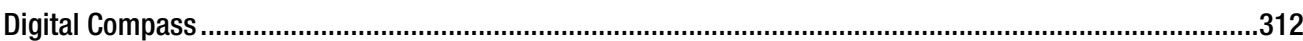

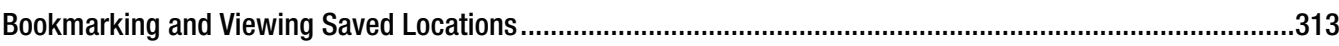

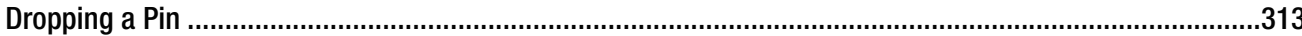

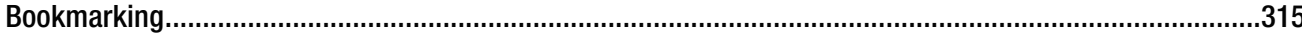

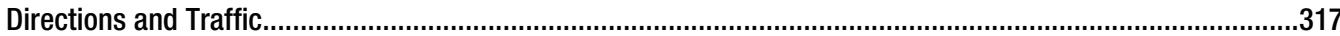

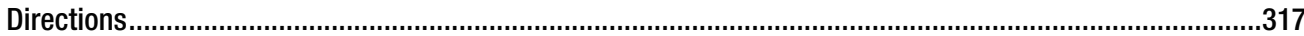

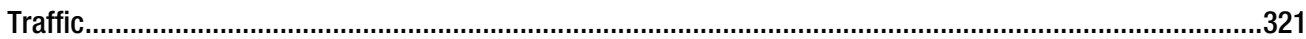

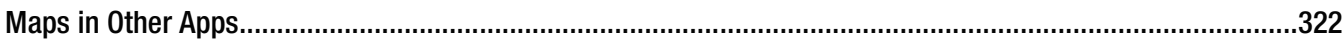

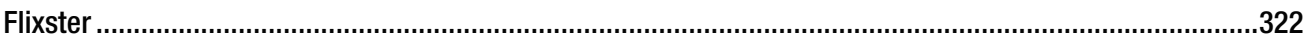

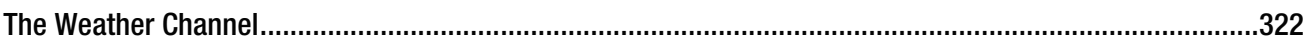

Ndrive US HD

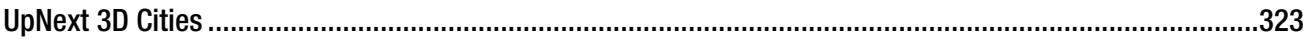

Find a Lost iPad

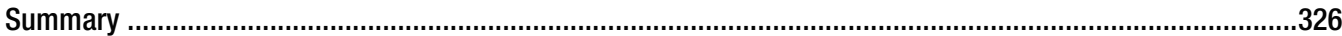

Chapter 13: Touching Your Digital Photos.................................................. 327

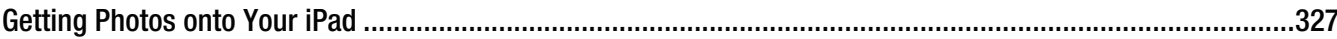

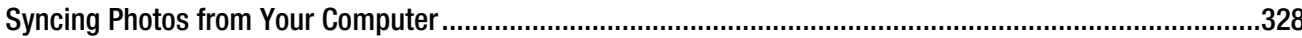

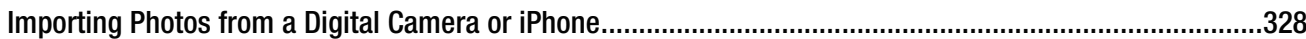

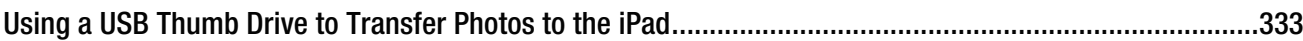

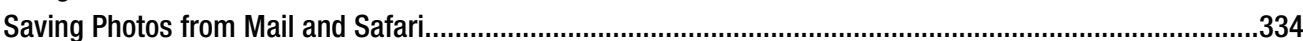

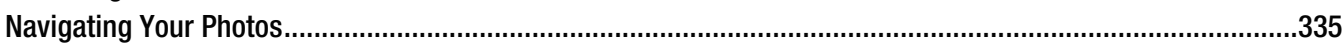

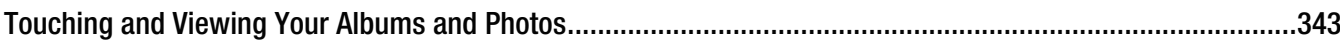

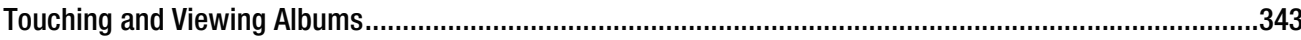

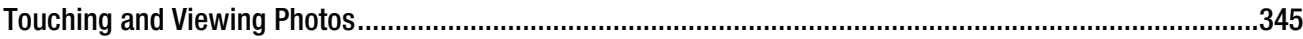

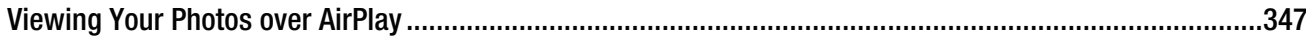

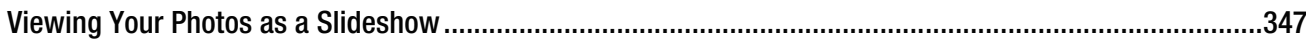

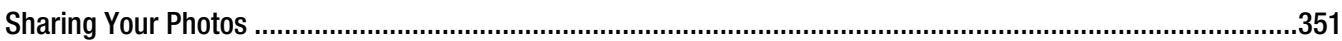

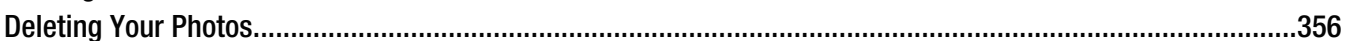

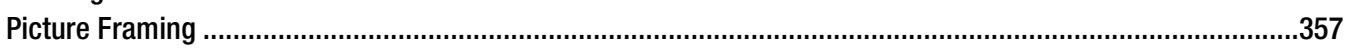

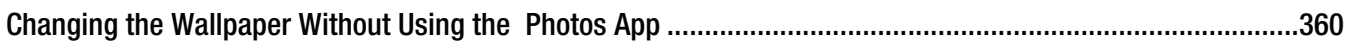

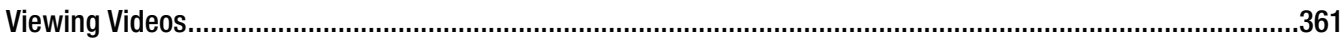

Editing Your Video

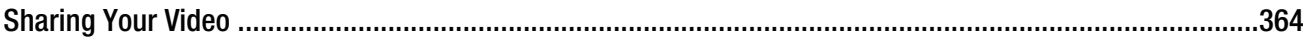

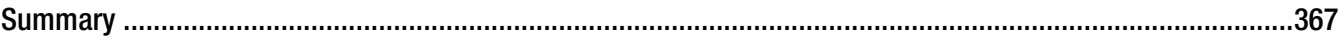

Chapter 14: On the Go with iWork ............................................................ 369

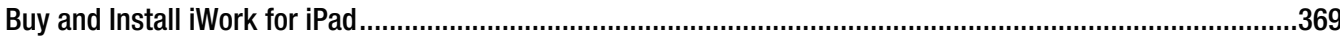

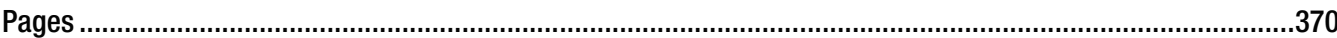

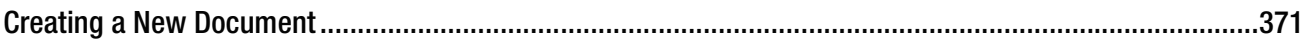




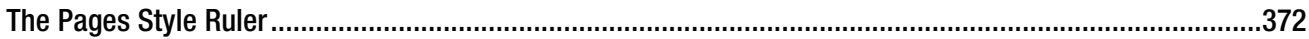

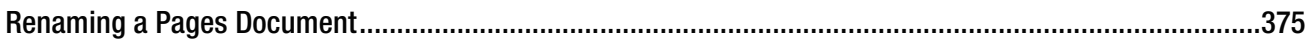

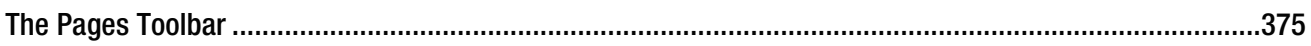

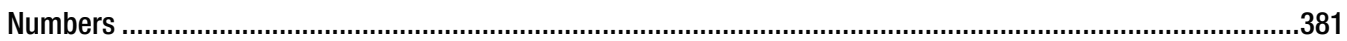

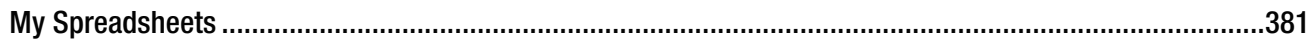

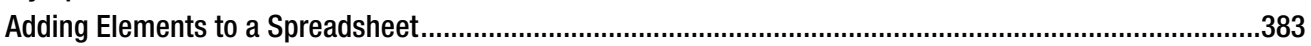

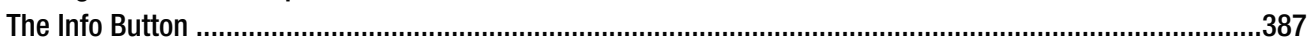

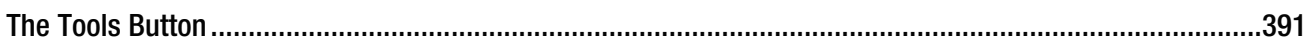

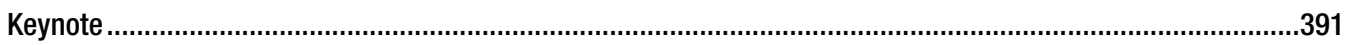

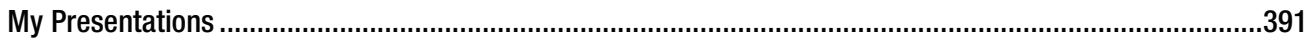

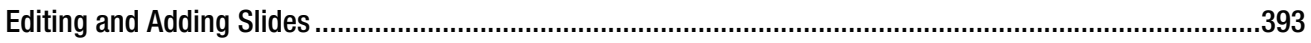

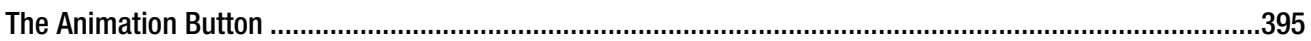

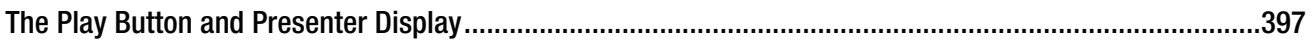

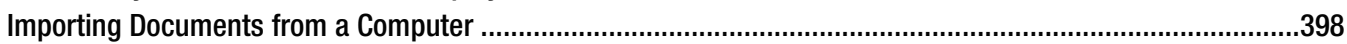

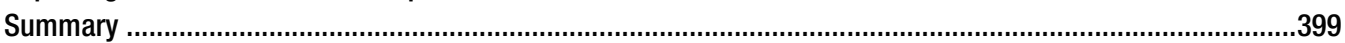

Chapter 15: Using the iPad Cameras with Camera, Photobooth, and FaceTime ....................................................... 401

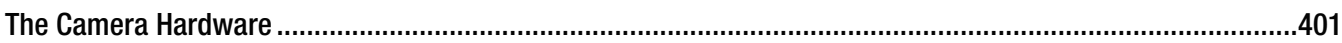

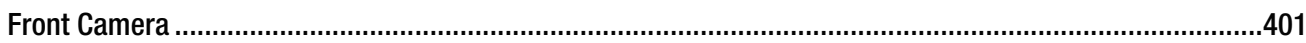

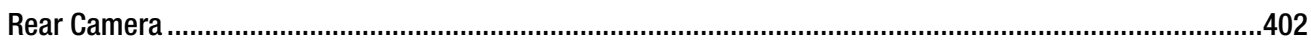

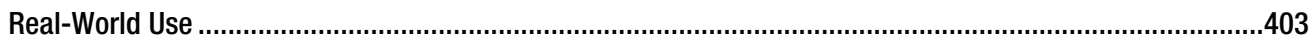

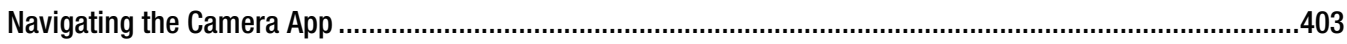

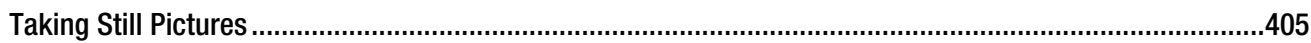

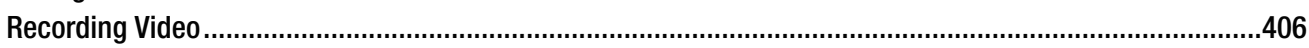

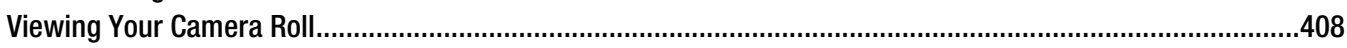

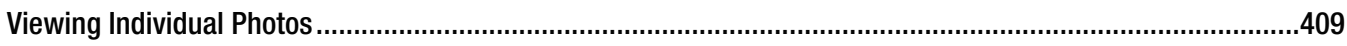

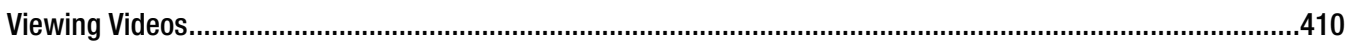

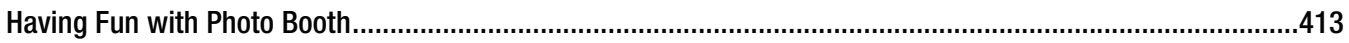

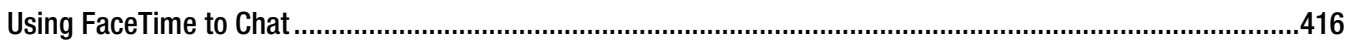

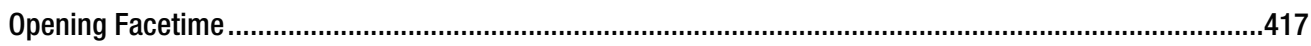

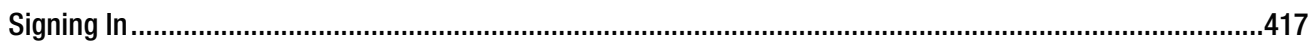

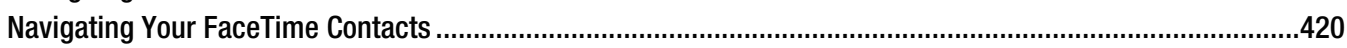

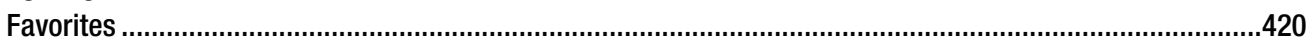

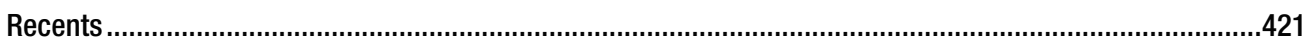

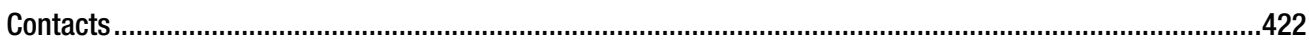

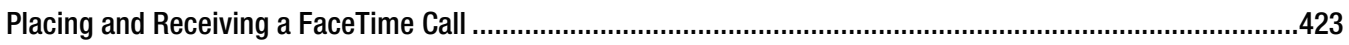

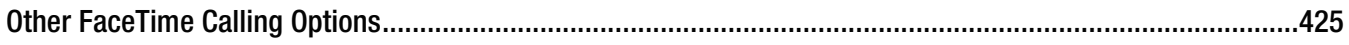

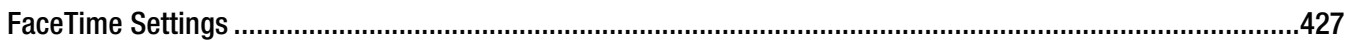

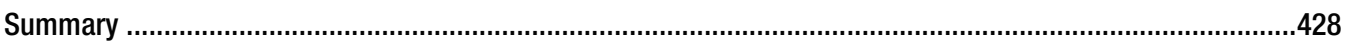

Chapter 16: Other Great Ways to Use Your iPad........................................ 431

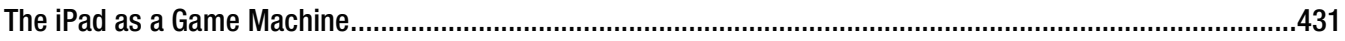

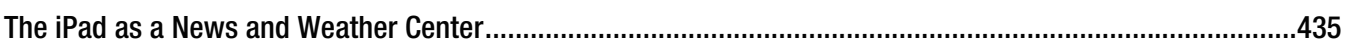

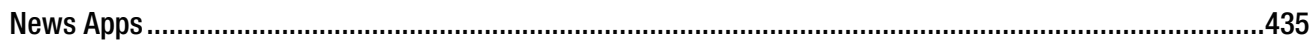

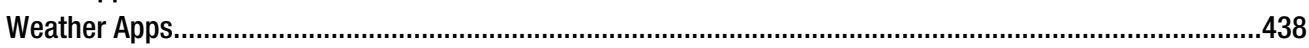

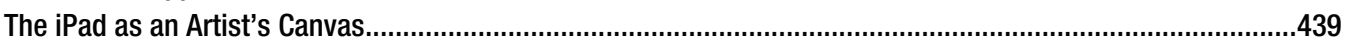

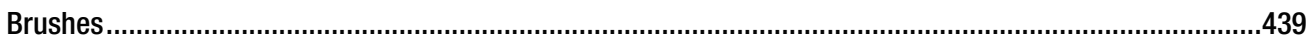

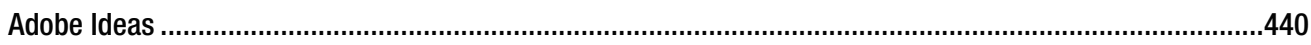




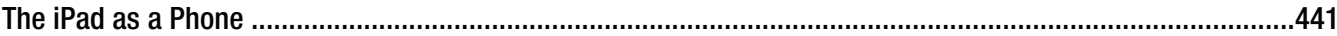

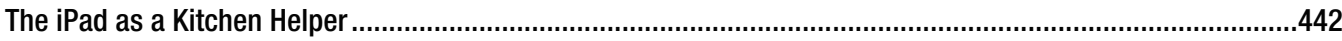

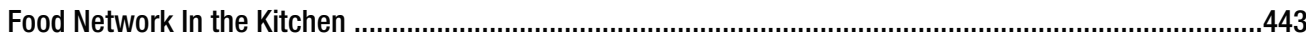

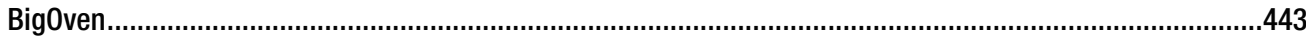

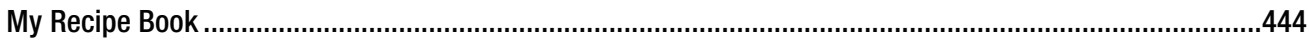

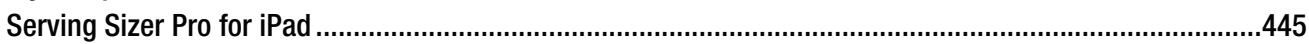

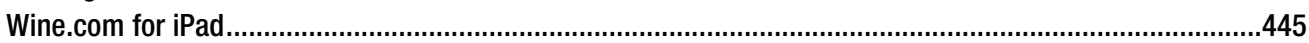

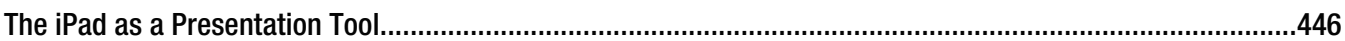

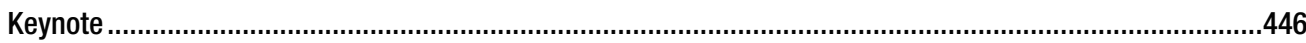

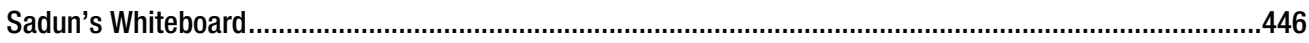

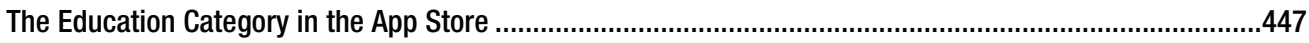

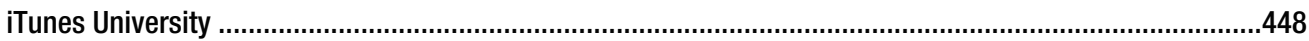

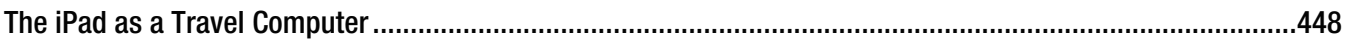

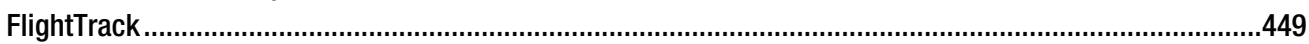

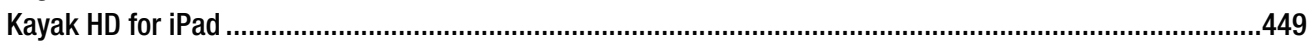

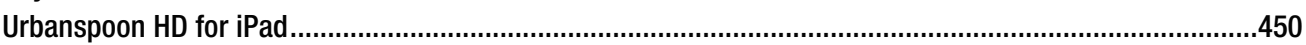

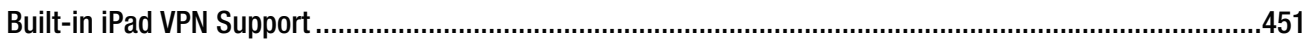

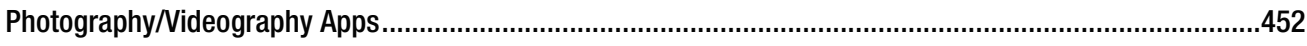

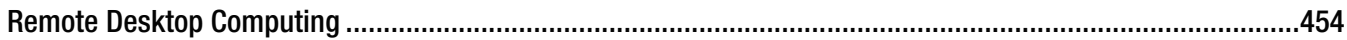

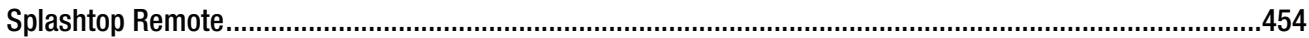

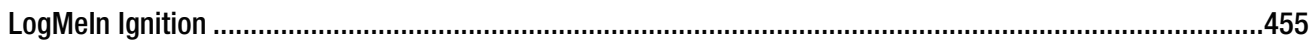

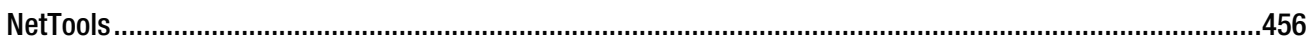

Summary

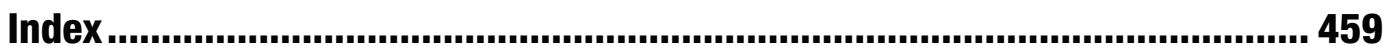




\section{About the Authors}

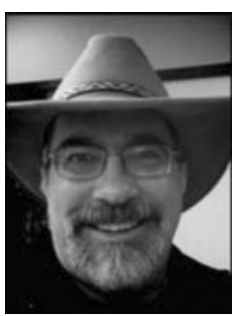

Steve Sande has been a loyal fan of Apple technology since buying his first Mac in 1984. Originally trained as a civil engineer, Steve's career as an IT professional blossomed in the 1990s. A longtime blogger, Steve is the features editor at AOL's The Unofficial Apple Weblog (TUAW.com), the author of three books about Apple's iWeb application, a collaborator on Taking Your iPad to the Max and Taking Your iPhone 4 to the Max, and working on the upcoming Taking Mac OS X 10.7 Lion to the Max. You can join Steve every Wednesday for the popular TUAW TV Live show, and follow his exploits at

www.twitter.com/stevensande. He lives with his wife of 32 years in Highlands Ranch, Colorado.

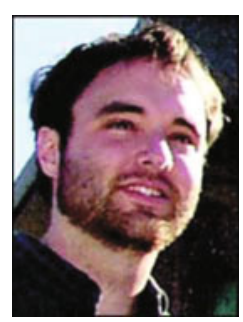

Michael Grothaus is an American novelist and journalist living in London. He was first introduced to Apple computers in film school and went on to use them for years to create award-winning films. However, after discovering many of Hollywood's dirty little secrets while working for 20th Century Fox, he left and spent five years with Apple as a consultant. He's since moved to London and earned his MA in Creative Writing. His first novel, Epiphany Jones, is a story about trafficking and America's addiction to celebrity. Currently, Michael is a staff writer at AOL's popular tech news site The Unofficial Apple Weblog (TUAW.com), where he writes about all things Mac. Additionally, Michael has written several other books for Apress, including Taking Your iPad to the Max, Taking Your iPod touch to the Max, 2nd edition, and Taking Your iPhoto' 11 to the Max. When not writing, Michael spends his time traveling Europe, Northern Africa, and Asia. You can reach him at www.michaelgrothaus.com and www. twitter.com/michaelgrothaus.

Erica Sadun holds a PhD in computer science from the Georgia Institute of Technology. She has written, cowritten, and contributed to about three dozen books about technology, particularly in the areas of programming, digital video, and digital photography. An unrepentant geek, Sadun has never met a gadget she didn't need. Her checkered past includes run-ins with iOS, Mac OS X, NeXT, Newton, and a vast myriad of both successful and unsuccessful technologies. When not writing, she and her geek husband parent three adorable geeks-in-training, who regard their parents with restrained bemusement. 


\section{About the Technical Reviewer}

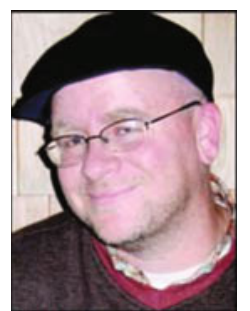

Dave Caolo is the Managing Editor at The Unofficial Apple Weblog, TUAW.com. He previously spent 8 years as the IT Director at a Mac-friendly residential school. When not geeking out with his Macs, Dave enjoys spending time with his kids, wife, and Boston Terrier, Batgirl. 


\section{Acknowledgments}

Thanks to everyone at Apress and TUAW. Also thanks to Steve Jobs for making such cool products. Let us add a special thanks to everyone who has bought this book. There are a lot of iOS books out there and we really appreciate that you've chosen ours. 


\section{Introduction}

Oh, how far we've come.

As we progress into the second decade of the 21st century, it's becoming increasingly obvious how the computers of the previous 30 years have begun to look not just old but archaic. The massive CRT monitors of the 1980s and 1990s have given way to pencil-thin displays of the 2000s. The PC's bulky and heavy beige box has been reduced to the size of a thick paperback, as with Apple's Mac Mini, or has even been assimilated into the display itself, as with Apple's iMac. This shrinking of the PC has also coincided with the computer becoming thousands of times more powerful-progress that shows no sign of abating. But although the miniaturizing and the explosive growth in processing power are technological accomplishments that are nothing to scoff at, the iPad signals the beginning of a new era in technology-the era of the touch-based computer.

When you hold the iPad 2, you hold the future in your hands. Its gesture-based controls have broken open the computer's digital/physical barrier. With the iPad 2, you can touch your movies, e-mail, and digital pictures; you can carry 10,000 books without increasing the weight in your bag; and you can flip through their pages with the swipe of your finger. Even the Web, something that has long been confined to a rigid desktop venue, becomes something akin to the magazine on your coffee table, yet with an infinite number of pages.

When the first iPad was originally introduced, some said it was nothing more than a big iPhone. Indeed, the iPhone introduced the world to the Multi-Touch interface, and both devices do use the same operating system called iOS. However, as you'll see, there are many minor and quite a few major differences. Using the iPad, your eyes are immediately enveloped by its large display; there's no squinting when you play games, navigate maps, and watch videos-all from your lap. Its full-size keyboard allows you to comfortably create documents, spreadsheets, and presentations with applications that rival those you find on older, traditional computers. No, the iPad isn't an over-grown iPhone; it's an evolution of the personal computer. The iPad 2 further defines the future of computers, making the device not just for consumption of media, but for its creation. Apple has added front- and rear-facing cameras for FaceTime video chatting and highdefinition video recording. It's now possible to not only listen to music, but create your own using the optional GarageBand software. Even video editing right on your touch screen is now a reality with Apple's iMovie for iPad.

Taking your iPad 2 to the Max introduces you to the iPad 2. Starting with helping you choose the iPad that's right for you, we walk you through purchase decisions and setting it up for the first time. You'll learn the gesture-based, Multi-Touch vocabulary that allows you to manipulate the $\mathrm{iPad}$ and its tens of thousands of apps. We'll show you how to connect to the Internet, browse the Web, touch your music and videos, and find and download apps from the App Store. You'll discover how to buy and navigate books using Apple's revolutionary iBooks app, view slideshows and photos, send e-mail, create notes and calendars, and even turn your iPad into a compass. You'll also discover that the iPad is not simply a new leisure device; it's a powerful office tool, and indeed can be a viable alternative to a traditional computer for many people. We walk you through creating rich documents, spreadsheets, and presentations. We also show you how to use the front- and rear-facing cameras to videoconference with friends and family and to even record 
your own movies. Finally, we show you some of our favorite third-party apps and ways we use the iPad as an artist's canvas, a teaching tool, and even a helper in the kitchen.

This book is written for anyone with an iPad 2 or anyone who is thinking of getting one (you won't be sorry!). It doesn't matter whether you're a Mac or Windows user or, in fact, if you've never used a computer before. This book's thorough coverage and step-by-step discussions allow all iPad 2 owners to learn about their device and come away with both the skills and the knowledge they need to use it to its fullest. If you owned an original iPad and just upgraded to an iPad 2, this book will still be of great benefit to you. The iPad 2 introduced many new hardware and software features that we cover thoroughly in this book.

How you read the book is up to you. If you are totally new to the iPad (or computers in general), we suggest you read the book cover to cover, but you can also feel free to jump around from chapter to chapter if that works better for you. Above all, have fun while you're learning everything that the iPad can do. It's the future of computing, and it's a blast. Thanks for letting us show it to you. 